\title{
A Spatially Heterogeneous Expert Based (SHEB) Urban Growth Model Using Model Regionalization
}

\author{
Dimitrios Triantakonstantis, Giorgos Mountrakis, Jida Wang \\ Department of Environmental Resources Engineering, State University of New York College of Environmental Science \\ and Forestry, Syracuse, USA \\ E-mail:dim30@aua.gr,gm@esf.edu,gdbruins@ucla.edu \\ Received March 26, 2011; revised May 13, 2011; accepted May 25, 2011
}

\begin{abstract}
Urbanization changes have been widely examined and numerous urban growth models have been proposed. We introduce an alternative urban growth model specifically designed to incorporate spatial heterogeneity in urban growth models. Instead of applying a single method to the entire study area, we segment the study area into different regions and apply targeted algorithms in each subregion. The working hypothesis is that the integration of appropriately selected region-specific models will outperform a globally applied model as it will incorporate further spatial heterogeneity. We examine urban land use changes in Denver, Colorado. Two land use maps from different time snapshots (1977 and 1997) are used to detect the urban land use changes, and 23 explanatory factors are produced to model urbanization. The proposed Spatially Heterogeneous Expert Based (SHEB) model tested decision trees as the underlying modeling algorithm, applying them in different subregions. In this paper the segmentation tested is the division of the entire area into interior and exterior urban areas. Interior urban areas are those situated within dense urbanized structures, while exterior urban areas are outside of these structures. Obtained results on this model regionalization technique indicate that targeted local models produce improved results in terms of Kappa, accuracy percentage and multi-scale performance. The model superiority is also confirmed by model pairwise comparisons using t-tests. The segmentation criterion of interior/exterior selection may not only capture specific characteristics on spatial and morphological properties, but also socioeconomic factors which may implicitly be present in these spatial representations. The usage of interior and exterior subregions in the present study acts as a proof of concept. Other spatial heterogeneity indicators, for example landscape, socioeconomic and political boundaries could act as the basis for improved local segmentations.
\end{abstract}

Keywords: Urban Growth Models, Spatial Heterogeneity, Model Fusion, Decision Trees, Denver

\section{Introduction}

Urbanization is a phenomenon observed since ancient times. It has been strengthened and acquired global magnitude over the last two centuries. More specifically, in year 1800 only $2 \%$ of people lived in cities, while in year 1900 the ratio increased to $12 \%$. In year 2008 , more than $50 \%$ of the world population lived in urban areas [1], and it is estimated that by year 2025 80\% of human population will live in cities [2]. This transition has and will change further socioeconomic structure, environmental resource allocation and ecosystem behavior. Urban environmental planning has been quantitatively and qualitatively supported by applying weighted overlay methods to the driving factors [3], as well as geostatistical techniques as an important part of the GIS-SPRING software capabilities [4]. It is therefore crucial to develop models for urban growth prediction to support interdisciplinary policy decisions for a sustainable future.

Numerous models have been recently developed for land use change prediction (for example [5-9]). The influence of biophysical and socioeconomic factors on land use changes has been an important issue in scientific debates [10] and significant investments are made in the understanding of linkages between ecosystems, climate and land use. For example, the National Science Foundation currently invests $\$ 22.5$ million to human-environment research, with a significant portion devoted to land 
use models [11].

Typically, land use models examine the likelihood for an area to be transformed from one land type to another [12]. Using available biophysical and socioeconomic variables as driving forces, approaches like linear/logistic regression, and heuristic methods of multicriteria evaluation can be adopted [13-15]. Logistic regression is a special case of generalized linear model, which is used to predict probabilities for the presence or the absence of a specific geographic characteristic. It has been widely used in urbanization [16-18]. In [19] logistic regression was used in order to predict urban-rural land conversion in a multi-temporal environment. Moreover, autologistic regression models have been developed in order to handle spatial autocorrelation. An additional explanatory variable, named autocovariate term can be applied to the logistic regression equation to correct the effect of spatial autocorrelation in a given neighborhood [20-22]. An alternative to the inclusion of spatial autocorrelation in the model expression is the introduction of an optimal sampling scheme to eliminate the spatial autocorrelation within the distance it occurs $[19,23]$.

Other models use fuzzy set theory as a method for dealing with imprecision of the data and determination of class boundaries [24-26]. Algorithms such as support vector machines [27-29] have been successfully applied to land use change modeling. Neighborhood effects are a major factor of land use dynamics [17,30-34] and an important component in many land use change models. The most common method to implement neighborhood interactions in land use change models is cellular automata [35,36], where the transition of a cell from one land use to another depends on the land use of its neighboring cells [37-40].

Artificial Neural Networks (ANNs) model complex relationships between variables, playing an important role as a non parametric approach in land use modeling [41-43] and land use change modeling [44-46]. In [47] a Land Transformation Model was successfully developed where social, political and environmental factors were examined to predict urbanization. This model was further used to forecast land use from 2000 to 2020 and the assessment was achieved using alternative drivers of land use such as forest species [48]. Another approach for future prediction of urban growth has been presented in [49], where the ART-MMAP, a neural network model, produces a prediction map under different scenarios related to historical urban growth data, land use drivers and socioeconomic data. ANNs have been also used for calibration and simulation of cellular automata models in urban systems [50,51]. ANN-based cellular automata models were also proposed for categorizing the cell transition in a binary way (urban/non urban) [52,53]. More- over, in [54] a generalized approach was introduced for multiple urban uses simulations (e.g. residential, commercial, and industrial).

Decision tree is another non-parametric learning algorithm widely used in land use/land cover modeling [55-59]. Structurally, it differentiates discrete instances, e.g. urban land use categories, through sequentially sorting down a bottom-up tree from the root/upper to the leaf/lower nodes. Each node represents a targeted attribute whose value is determined by a partitioning rule associated to the branch descending from the upper-level node [60]. Compared to generalized linear models, a decision tree is more robust to data distribution such as outliers or missing values, and more flexible in establishing rules that are spatially heterogeneous [61]. Compared to other non-parametric approaches such as ANNs, rules established by decision trees are structurally simple and readily interpretable [55]. However, traditional decision trees treat data as a collection of independent observations, and thus exclude the influence of data spatial autocorrelation in the training process. This limitation has been investigated by a spatial entry-based decision tree designed by [56] where a notion of "spatial entropy" was proposed.

An important aspect of urbanization is spatial heterogeneity [62]. It was soon realized that similar values in an explanatory variable may have different effects in the urban development of different areas and therefore must be treated separately. Although a decision tree universally incorporates a higher degree of spatial heterogeneity, the robustness is yet limited by its intrinsic single-algorithm structure where the complete area is indiscriminately targeted into a global rule [63,64]. Classification and regression trees were used to divide a forested area into homogeneous parts in order to localize the global model [65].

The urban spatial structure and change dynamics can be better described by applying spatial metrics [58,66-68]. Spatial metrics describe spatial heterogeneity by dividing large areas into homogenous subregions. Examples of metrics used to quantify the spatial heterogeneity include patch size, patch density, edge length, distance from nearest neighbor and contagion among others [69]. Moreover, the fractal dimension is also implemented as a spatial metric to describe patch complexity [70,71]. In [72] a region-based system was developed to deal with the spatial and morphologic characteristics of urban structures. Spatial heterogeneity also exhibits scale dependency [73,74]. In [75] a clustering approach was used to map urban influence at multiple scales; the micro, meso and macro scales have been a useful foundation for exploring spatial dynamics of urban structure, addressing spatial, temporal and behavioral complexity 
[11].

This paper investigates whether integration of spatially unique models improves on capturing spatial heterogeneity. We investigate whether an expert-based selection of multiple models operating in different spatial regions outperforms a global model with the same input variables (including the segmentation variables) using an identical training dataset. Our implementation includes decision tree classifier and variable training data sizes on a binary urbanization prediction task.

\section{Study Area and Modeling Data}

\subsection{Study Area}

The study area is located in the Denver metropolitan area, Colorado, which is in the center of the Front Range Urban Corridor, with the Rocky Mountains from the west and the High Plains from the east. The area selected for this study covers the major part of Denver metropolitan area and is specified by $X_{\min }: 481862 m, X_{\max }: 522032 m$ and $Y_{\min }: 4389809 m$ and $Y_{\max }: 4421313 m$ (UTM Zone 13 North), as Figure 1 shows. Denver has experienced a large urban growth from 1977 to 1997. According to land use maps, provided by the U.S. Geological Survey Rocky Mountain Mapping Centre

(http://rockyweb.cr.usgs.gov/frontrange/datasets.htm), the percentage of urban growth from 1977 to 1997 was 20.8\% (urban areas in 1977: $48 \%$ and 1997: $58 \%$ of the total study area). This rapid urban growth was the motivation behind this site selection for our model development.

\subsection{Response and Predictor Variables}

The urban development is the response variable in this current study. The non-developed areas in 1977 that are converted to developed areas in 1997 are assigned as 1 into the response variable, while the non-developed areas 1977 which remain the same in 1997 are given the 0 value. The developed areas in 1977 are excluded from the model and we also assume no conversion from developed back to non-developed area. The urban developed areas include residential areas, commercial/light industries, institutions, communication and utilities, heavy industries, entertainments/recreations, roads and

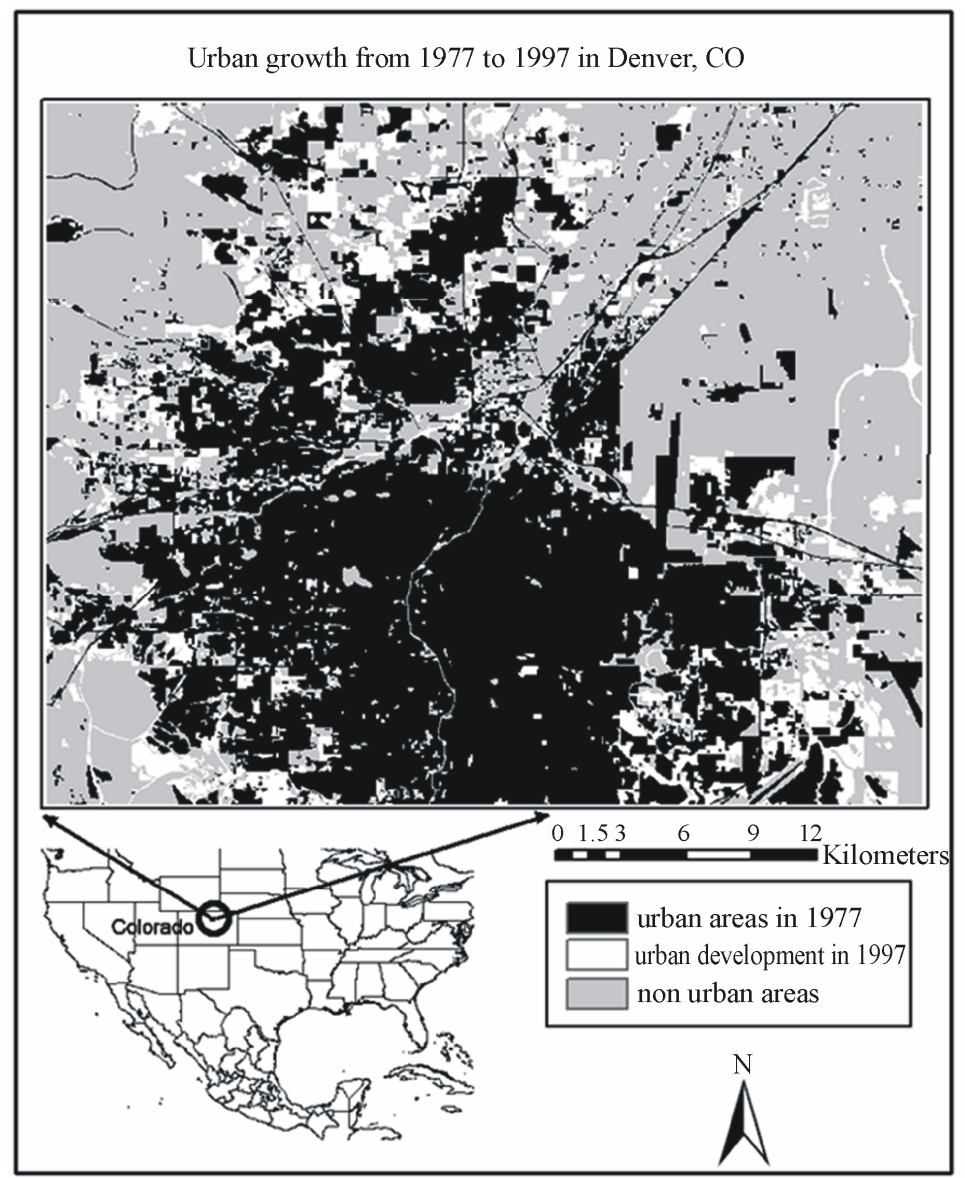

Figure 1. Urbanization changes in the Denver, CO metropolitan area. 
other transportation.

We examine 21 predictor variables which are produced using Euclidean distances to the nearest neighbor and Kernel density filters. The predictor variables include: a) Euclidean distance to entertainment venues, heavy industries, rivers, primary roads, secondary roads and minor roads, b) Kernel density (radius: 120 pixel) of agricultural business, residential areas, urban developments, commercial areas, institutes/schools, communications/utilities, lands/ponds, cultivated lands and natural vegetations, c) Kernel density (radius: 10, 30, 50, 80, 100, $150)$ of distance to urban developments. All the aforementioned variables were based on 1977 vector data, no information from 1997 was incorporated as that was our prediction year. Furthermore, elevation and slope are also considered, making 23 the total number of predictor variables. Statistical analysis in this study area shows that distance to entertainment, density of residential areas, density of urban development and density of natural vegetations contribute with higher importance in modeling the urban growth than the other predictor variables [68]. The final form of the dataset expressing response and predictor variables is in a raster representation with a $30 \mathrm{~m}$ spatial resolution.

\section{Model Development}

\subsection{Theoretical Underpinnings}

Several algorithms have been proposed for urban modeling with varying complexity and success. A motivating factor behind algorithmic selection relies on an algorithm's ability to capture spatial heterogeneity. The current approach is to rely solely on algorithmic complexity to adjust model behavior in different regions of the entire study site. In this paper we examine whether a segmentation of the study area in subregions followed by selective application of methods within each subregion would lead to improved modeling capabilities. In other words through model regionalization we challenge the current expectation that a highly complex globally applied method can sufficiently recognize local heterogeneity and fine tune performance accordingly.

From the model development perspective, we train different models in different subregions and then spatially group the results obtained. These subregions are identified based on expert knowledge on different urbanization drivers. In order to allow a global model to directly compete with our numerous local models the segmentation criterion used to define subregions is also incorporated as an additional input variable to the global model. Therefore the global model has equal opportunity to capture heterogeneity as the local models, because the same input variables and the same modeling techniques are implemented in both cases.

We apply decisions trees in order to evaluate our hypothesis of multiple local models outperforming a global one. Decision trees are a popular modeling technique as in addition to advanced modeling capabilities, they still remain easy to understand as they can be converted to a set of rules. Decision trees use a training dataset in order to construct the model structure and the produced model is applied to a different dataset (validation) to estimate the prediction accuracy. Of particular interest is performance assessment of local vs. global models on a varying training dataset size. Small training sizes are cost-efficient to acquire but there is an overfitting cost associated with them, therefore the identification of proper balance is investigated.

\subsection{Subregion Identification Based on Heterogeneous Behavior}

Extraction of homogenous areas is typically based on fragmentation analysis where spatial and landscape metrics are adopted. A wide range of relevant metrics has been proposed, especially in ecological applications [67]. In our case fragmentation analysis involved evaluation of spatial distribution of urban development in the entire study area. It was found that some areas have higher propensity for urban development than others; a consequence of urbanization density. More specifically, an area surrounded by urban structures may experience different development pressures than not surrounded areas [68].

In our study, the entire area is divided into two subregions: the interior urban subregion, a dense urbanized area and the exterior urban subregion with no dense urban structures (Figure 2). These two areas exhibit different propensity for urban development.

Figure 3 demonstrates the cumulative probability that an undeveloped pixel in 1977 would be developed in 1997 at a given distance. That relationship is clearly different for the interior and exterior subregions at various distances from already developed areas, which were measured using Euclidean distances between pixel centers. Note that the intention of this graph is to provide a relative comparison between exterior and interior regions leading to motivation behind the model development; the graphs purpose is not to directly incorporate these probabilities in model design. Undeveloped areas in close proximity to existing urban structures are more likely to be converted to urban land use in general, but note that this probability is significantly higher in interior subregions. This is mainly due to the intense human influence which occurs near existed urban structures in dense urban environments such as the interior subregion. For 


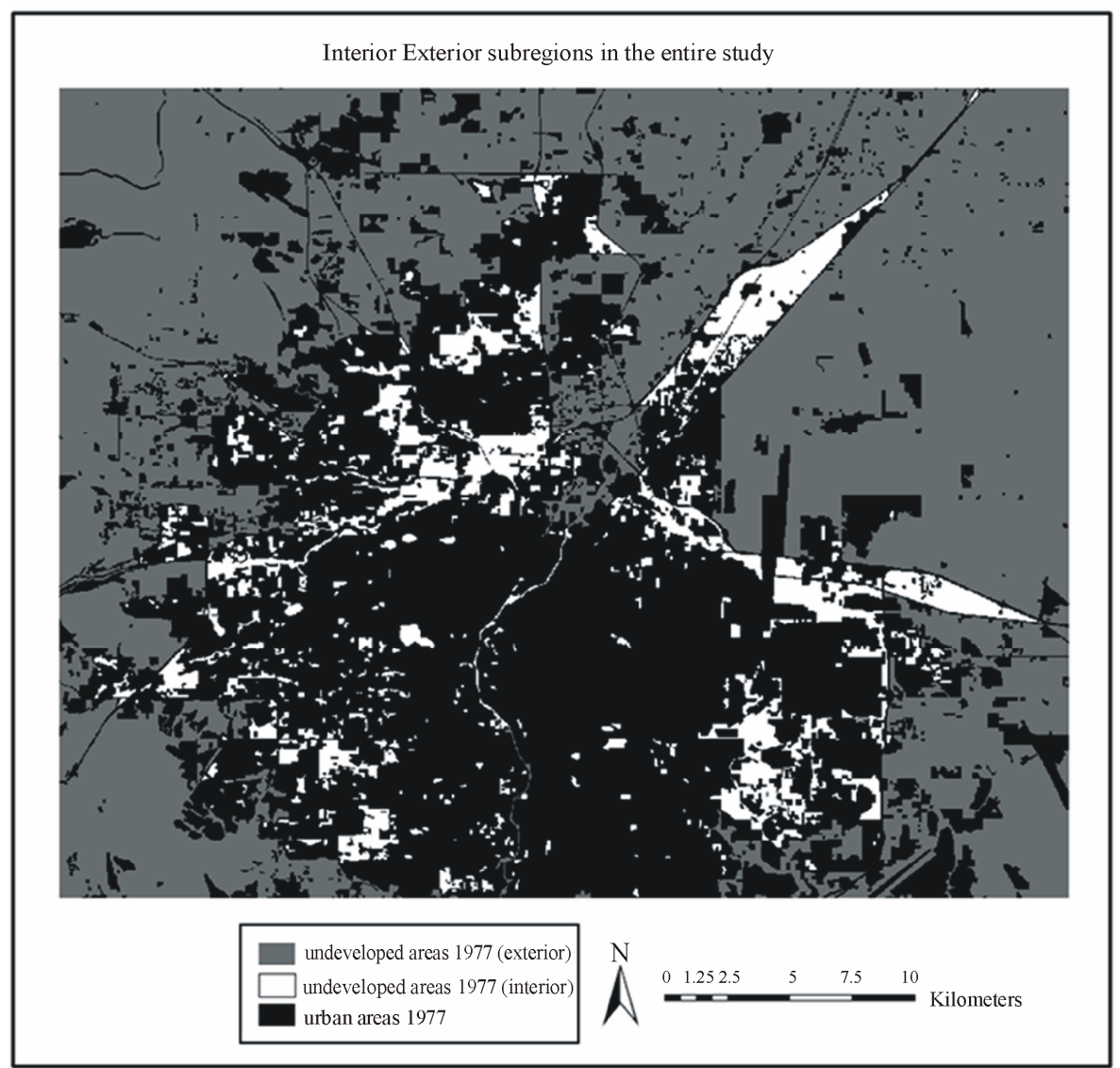

Figure 2. Interior and Exterior subregions of the 1977 Undeveloped area.

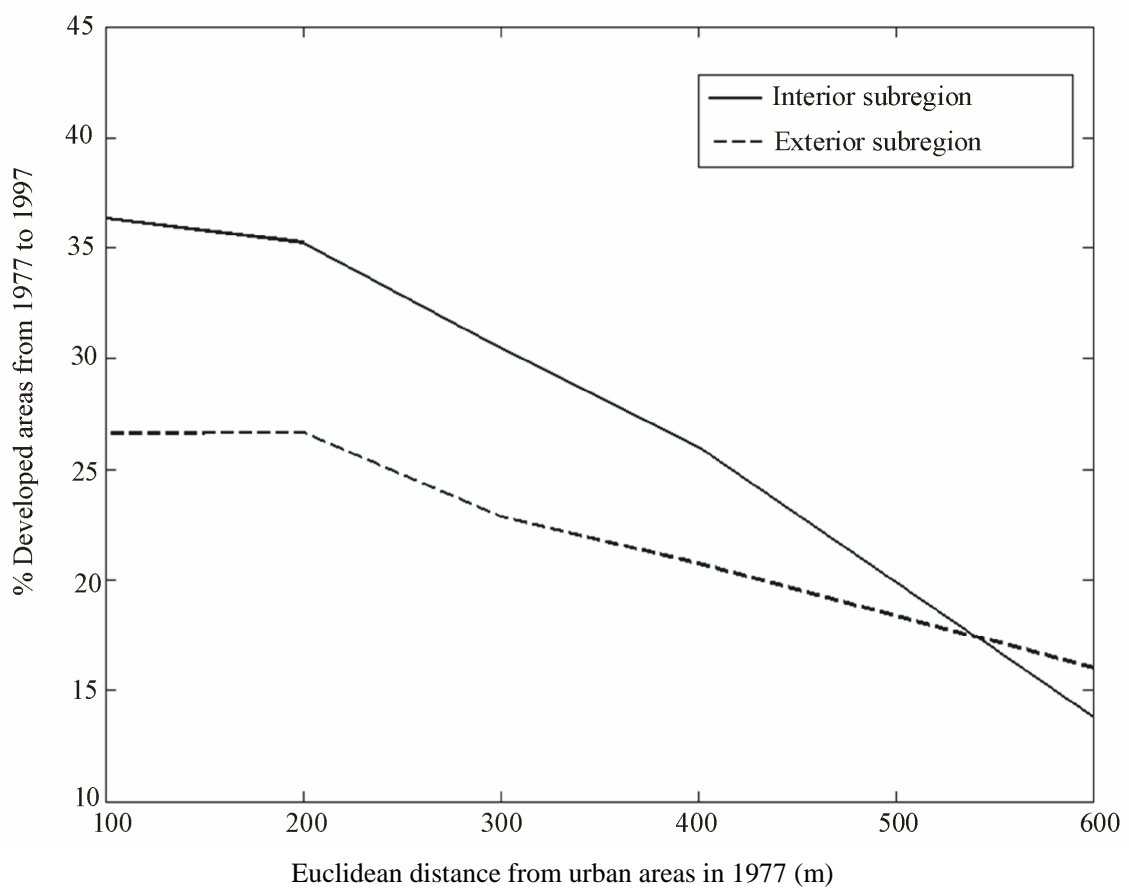

Figure 3. Development probability as a function of Euclidean proximity to existing urban structures for the interior and exterior subregions. 
example, the commercial value of these properties may be higher than places far away from buildings. Therefore, the decision to separate in the proposed models interior and exterior areas reflects expert knowledge on expected urban development behavior.

Motivated by the divergence in urban development behavior we develop the proposed local models for each subregion (one for the interior and another for the exterior) and contrast them with a global model trained and operating in both subregions simultaneously. Further segmentations are possible, especially for the exterior subregion, however this interesting investigation is reserved for future work. The purpose of this manuscript is to demonstrate the proof of concept on model regionalization and excite additional research.

\subsection{Model Design and Experimental Setup}

The proposed Spatially Heterogeneous Expert Based (SHEB) model uses multiple decision trees to capture urban growth. The entire study area is divided into the interior and exterior subregions leading to the creation of multiple models to test model regionalization benefits. If a model is trained using samples exclusively from a subregion it is called Local, if samples come from the entire study area the name Global is assigned. We also use a subscript index in the naming structure to reflect where the model is simulated for validation purposes, for example Global $_{\text {int }}$ relates to a globally trained model validated only in the interior subregion. All the Local models are trained and validated exclusively in the same subregion, therefore the notation Local ${ }_{\text {int }}$ for example suggests a local model trained and validated in the interior subregion. As a result of the above we have developed the following models:

- Local $l_{\text {int }}$ : training and validation dataset from the interior subregion.

- Local $_{\text {ext }}$ : training and validation dataset from the exterior subregion.

- Global int $_{\text {: }}$ training dataset from the entire study area, validation dataset only from the interior subregion.

- Global ${ }_{\text {ext }}$ : training dataset from the entire study area, validation dataset only from the exterior subregion.

- Global ${ }_{a l l}$ : training and validation dataset from the entire study area.

We should clarify that Global $i_{\text {int }}, G_{\text {Gobal }}$ ext and Global $_{\text {all }}$ are the exact same model since they are all produced from the same training set from the entire study area; however, model performance is validated in different regions to allow comparisons with the corresponding Local models.

In order to identify the optimal balance between Local and Global models we perform comparisons in each subregion (interior and exterior) and in the overall site (all region). The term balance is used to refer to the fact that not always Local models will outperform global ones; in every region we compare the corresponding Local with the Global model and decide which one to use. The subregion analysis lead to the following pairwise comparisons: a) Local $_{\text {int }}$ and Global $_{\text {int }}$, b) Local ext $_{\text {t }}$ and Global $_{\text {ext }}$. For each subregion, the comparison between the Local and Global models assesses whether spatial heterogeneity should be addressed separately in that region. Depending on the subregion accuracy assessment the predominant subregion-specific model is selected to participate further into the SHEB model structure. Since the $S H E B$ model expects to operate over the entire study site it is compared against the Global $_{\text {all }}$ model. These comparisons are presented graphically in Figure 4.

\subsection{Algorithmic Specifics}

The decision tree models were developed and evaluated in the Matlab environment. Ten observations were set as the minimum for a node to be split. Moreover, each decision tree is adjusted using a 10 -fold cross-validation and a pruning process.

In order to compare Local and Global models we had to ensure comparable model complexity and input selection. Regarding input selection the Local models contain the aforementioned 23 predictor variables (see section 2.2). The Global models incorporate the exact same 23 variables plus an additional predictor variable: a dummy variable with value of 1 if a point belongs to the interior subregion and the value of -1 if it lies in the exterior subregion. By doing so, the Global models have the potential to express the expert-derived interior/exterior segmentation within their model structure. In terms of model complexity the decision trees developed for Local and Global models are directly comparable because the training of each model took place considering the same minimum number of points (10 points) classified in every leaf.

The reference output variable is dichotomous, with value 1 if the change is from non-urban in 1977 to urban in 1997, and 0 when the 1977 non-urban areas do not change. The reference output binary variable is compared with the predicted output of SHEB and Global $_{\text {all }}$ models. Each set of predictor values is inserted into the decision tree, which is produced using the regression tree option in Matlab, and a corresponding response value is predicted. Because the reference response variable contains only two numeric values, 0 and 1 , the corresponding predicted output is a continuous variable with a range between 0 and 1 , indicating the probability for change. The closer the probability to 1 , the more likely 


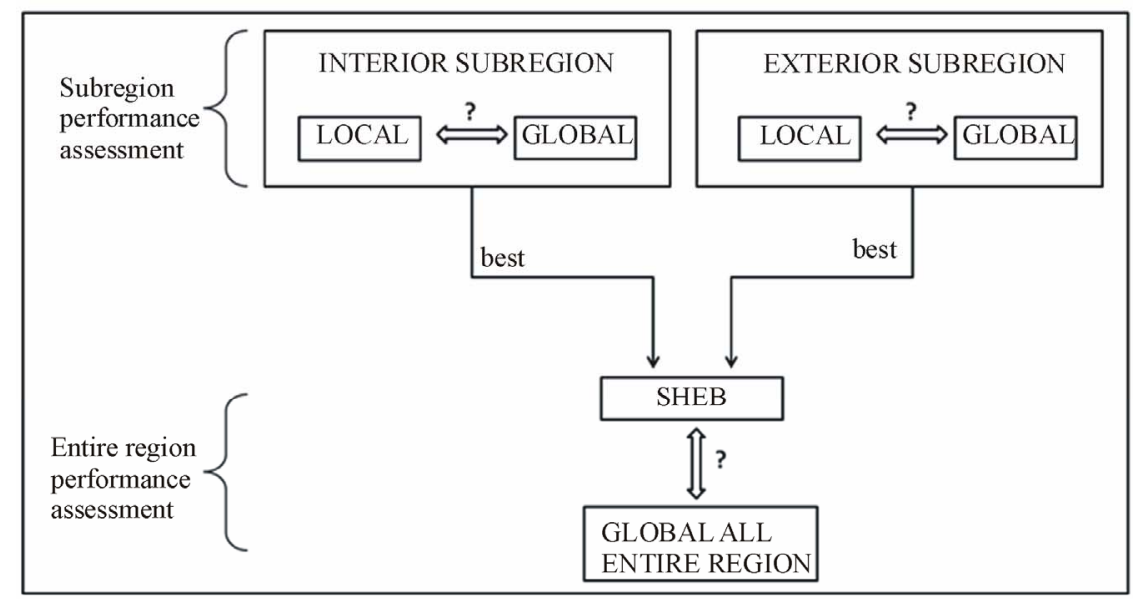

Figure 4. Design scheme of SHEB urban growth model.

this area is to experience urban development. A threshold is applied in order to categorize the values of the predicted output into two classes: 0 and 1 . In most cases, a 0.5 threshold is used, so as values greater than 0.5 to be classified to 1 (developed), otherwise to 0 (non-developed). This value of 0.5 was used as threshold in our study as well.

\subsection{Training Sample Specifics}

From the entire study area (710,536 points) we extracted $70 \%$ of the data points for validation purposes $(497,375$ points) and kept the remaining 30\% for various training experiments. The validation dataset contained 389,810 no change and 107,565 change points; spatially it was distributed to 59,755 interior points and 437,620 exterior points. All statistics reported in the results section are calculated using the same validation dataset.

We examined a variety of training sample sizes to assess model performance. We varied the training sample from 4000 to 30000 with an increment of 4000 leading to 14 different training sets. For a given training sample total size goal (e.g. 4000 total training points), we randomly selected equal number of interior and exterior training points (e.g. 2000 for each). Each Local model was trained with the corresponding points (e.g. the 2000 interior points for the Local $_{\text {int }}$ model) and the corresponding Global model used the identical points from the two Local models combined (e.g. the 2000 interior points for the Local $_{\text {int }}$ model and the 2000 exterior points for the Local $_{\text {ext }}$ model leading to the 4000 point training dataset for the Global model). Identical points were used to support direct comparison between Local and Global models. Furthermore, for each training dataset total size (e.g. 4000) we performed 50 random sampling selections to limit bias especially in smaller size datasets.

Semivariograms analysis showed that spatial autocor- relation exists within $450 \mathrm{~m}$. In order to overcome this difficulty, training sets were produced using several random samplings, all at least $450 \mathrm{~m}$ apart from each other. Because of the reduced number of training points, high overfitting occurred with large discrepancies between calibration and validation accuracies. Therefore, the spatial autocorrelation is not considered in this paper and any point could participate in model calibration/ validation.

\section{Results}

Results from each subregion are presented in the subregion performance assessment section. Using these results as a guide, a proposed SHEB model is created and contrasted with a Global model leading to the entire region performance assessment. The aggregation statistics in the entire region put equal weight to both interior and exterior subregions to avoid a site-dependence bias. We should note that the term prediction relates to the extrapolation on historical data at later times.

\subsection{Interior and Exterior Subregion Performance Assessment}

The performance of SHEB model is evaluated using the confusion matrix and the Kappa statistic. Confusion matrix is produced by cross-tabulation between predicted and actual variables [76,77]. It is the percentage of predicted cases which are correctly classified either as urban or non urban areas. Kappa statistic is a more robust method in classification accuracy, because it can provide concordance avoiding the cases which are correctly classified by chance [78].

In Figure 5 the accuracy results (Kappa, accuracy percentage) in the interior and exterior subregions are graphically displayed by boxplots. Each box contains the 
median value (central mark) and the 25th and 75th percentiles (edges of the box) for 50 random training sets. The graph presents pairs of local and global models and they are slightly offset for visualization purposes. Every pair of local-global is associated with a certain training sample size that is presented on the $\mathrm{X}$ axes. The training sets of SHEB models for each subregion (interior/exterior) vary from 2000 to 15000 points providing a
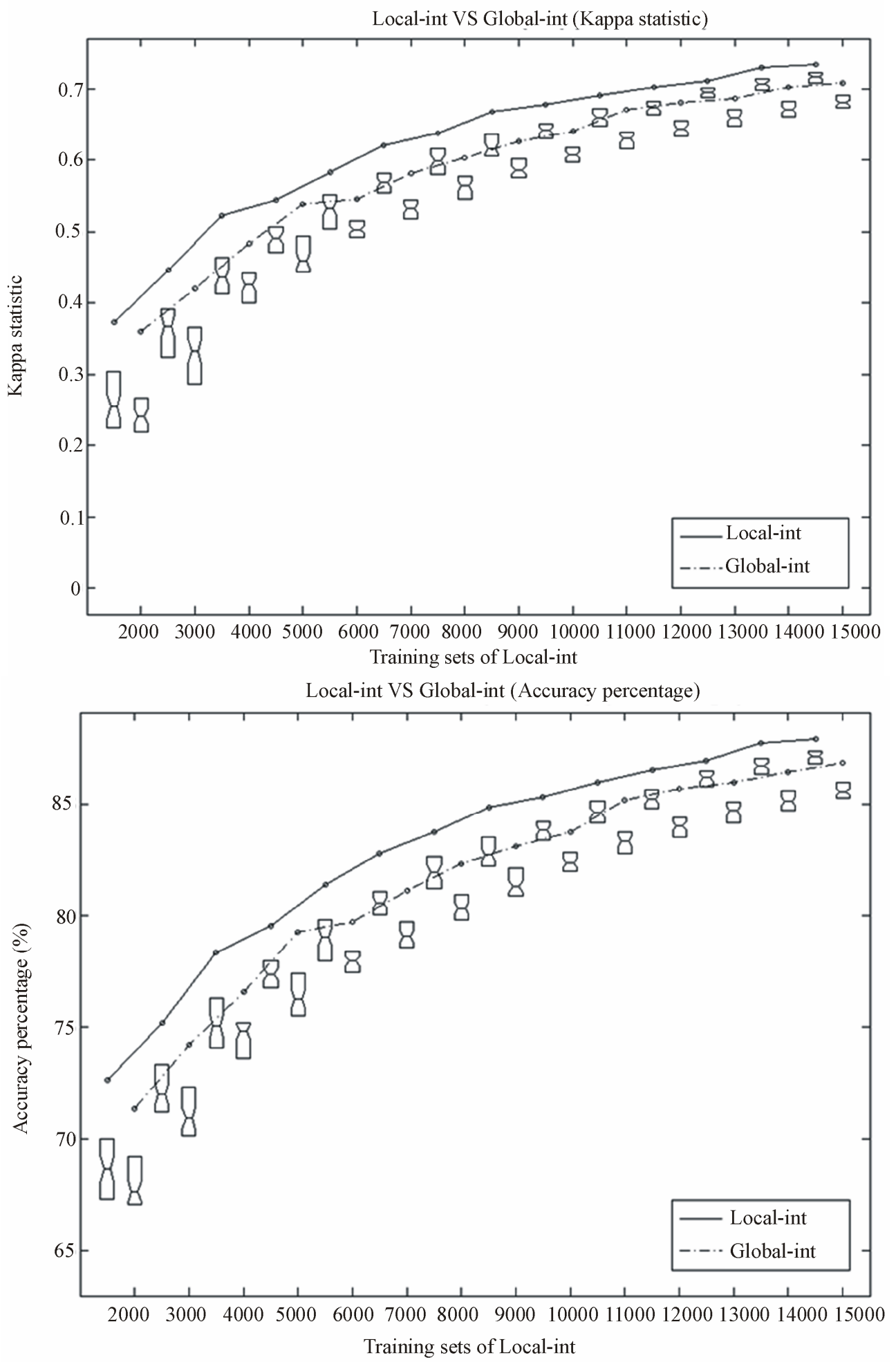

(a) 

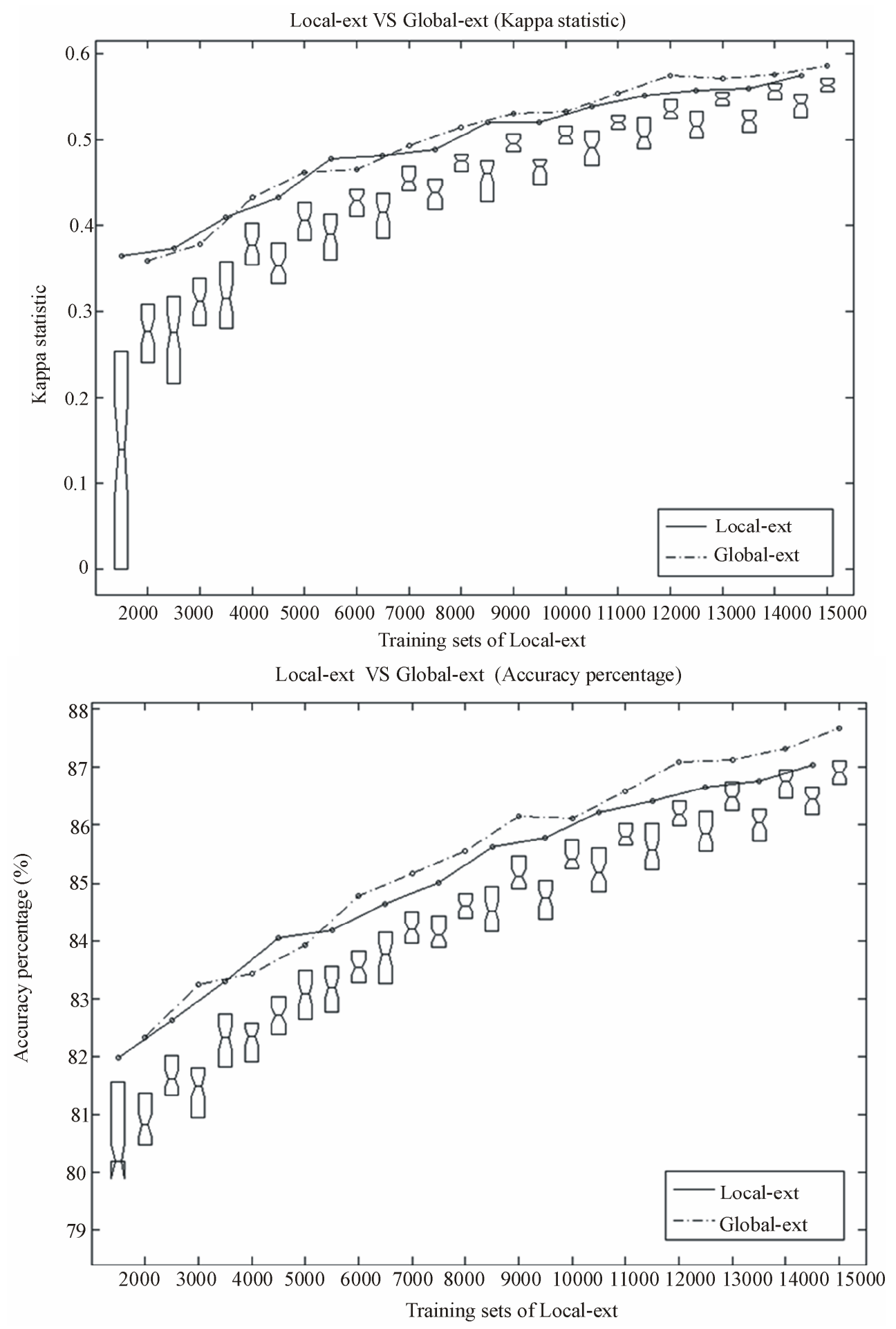

(b)

Figure 5. Comparison between Local and Global models using decision tree algorithms. (a) Decision trees assessment within the interior subregion; (b) Decision trees assessment within the exterior subregion.

total from 4000 to 30000 points; the exact same points are used in the corresponding Global models. Fifty different decision trees are produced for each training size.
This process minimizes the bias regarding the randomness of training set selection. Moreover, a line connecting the maximum value of Kappa and accuracy percent- 
age for each training size is drawn.

The comparison within the interior subregion using models Local ${ }_{\text {int }}$ and Global ${ }_{\text {int }}$ for both Kappa and accuracy percentage in different training sizes is given in Figure 5(a). The comparison in the exterior subregion between Local $_{\text {xxt }}$ and Global ${ }_{\text {ext }}$ is presented in Figure 5(b). In the interior subregion, the Local $_{\text {int }}$ model exhibits significant improvements over the Global $_{\text {int }}$, while the results for the exterior subregion do not show significant differences between the Local $_{\text {ext }}$ and Global ${ }_{\text {ext }}$ models.

\subsection{Entire Region Performance Assessment}

\subsubsection{Single Pixel Assessment}

Using the subregion performance assessment we fuse the Local Interior model (Local ${ }_{\text {int }}$ ) with the Global Exterior model (Global $e_{\text {ext }}$ ) to formulate the proposed SHEB model. The SHEB is then compared to a decision tree-based Global model (Global ${ }_{\text {all }}$ ) operating on the entire study area. Since both SHEB and Global model use the same model to classify points in the exterior subregion, algorithmic improvements are due to any performance differences in the interior subregion. We average improvements over both regions to produce the overall accuracy and Kappa statistics comparisons of Figure 6. It is worth mentioning that in decision tree graphs (Figures $\mathbf{5}$ and $\mathbf{6}$ ), both Kappa and accuracy percentage are sensitive to the number of training points, as expected. In order to examine the significance of Kappa statistic as well as the accuracy percentage in the two pairwise comparisons (SHEB VS Global $_{\text {all }}$ ), a paired Student's t-test is carried out. The comparison aggregates differences between the best two models for each training dataset size. This test is used to investigate performance relationship between two models, without considering any one-to-one correspondence between points belonging into the same group. According to Table 1, SHEB model differences from the Global model in terms of both Kappa and accuracy percentages are statistically significant $(\mathrm{a}=0.05)$. In addition, the negative $t$ values for the exterior subregion justify the selection of the Global over the Local model to participate in the SHEB model.

\subsubsection{Neighborhood Assessment}

The above accuracy metrics are based on a pixel per pixel comparison between model output and reference data. Multi-scale accuracies are also used to aggregate performance within a neighborhood moving away from individual pixels. The multi-scale accuracies capture the similarity of patterns providing an assessment in different resolutions. More specifically, the multi-scale accuracy assesses the number of changes occurred in a specified window versus the actual number of changes with- out taking into account the exact spatial specificity of these changes as long as they take place within a local neighborhood. This assessment technique is important and beneficial for potential users such as policy makers and planners, where algorithmic performance in a given window (e.g. a 1 mile block) is more desirable rather than the actual locations of urban sprawl within that neighborhood. The calculation is defined by the following formula [79].

$$
F_{i, d}=\frac{\sum_{i=1}^{n}\left|1-\frac{m_{i, d}-\bar{m}_{i, d}}{M_{i, d}}\right|}{n}
$$

where $F_{i, d}$ is the accuracy of the pixel $i$ in a window with $d$ diameter size of the circular window within which the accuracy is calculated, $m_{i, d}$ is the actual changes occurred in $d, \bar{m}_{i, d}$ is the predicted changes in $d, M_{i, d}$ is the total valid pixels (excluding the existing urban developed pixels in 1977) in the examined window and $n$ is the total population of pixels.

In Figure 7, the graphs of multi-scale accuracies are presented using the training sets of 4000, 16000 and 30000 points for both SHEB and Global ${ }_{\text {all }}$ models. For each training size test, the best algorithm of the 50 decision trees was selected in order to calculate the multi-scale accuracies. The size of neighborhood ranges from $3 \times 3$ to $70 x 70$ pixels (90m to $2100 \mathrm{~m}$ ). The fusion between Local $_{\text {int }}$ and Global $_{\text {ext }}$ (SHEB model) offers increased accuracy when compared to the Global ${ }_{\text {all }}$ model. For example, at the $1 \mathrm{~km}$ scale, a representative planning scale for the urban community, accuracy improvement varies from $1 \%$ to $3 \%$. Most importantly, improvements are more significant for smaller training set sizes, which makes the proposed method even more appealing considering that data availability is a typical limitation in such models.

\section{Discussion and Conclusions}

A Spatially Heterogeneous Expert Based (SHEB) model, which addresses spatial heterogeneity using multiple region-specific models, is introduced in this research. Our hypothesis investigates whether expert knowledge improves prediction accuracy through model regionalization, in other words whether the integration of different models in homogeneous local subregions outperforms a global model trained in the entire study area. Most similar studies capture the spatial heterogeneity using the differentiated factor, the factor which describes this dissimilarity, as an additional term to a global model applied in the entire study area. In contrast, the SHEB model uses this expert knowledge prior to the model ap- 

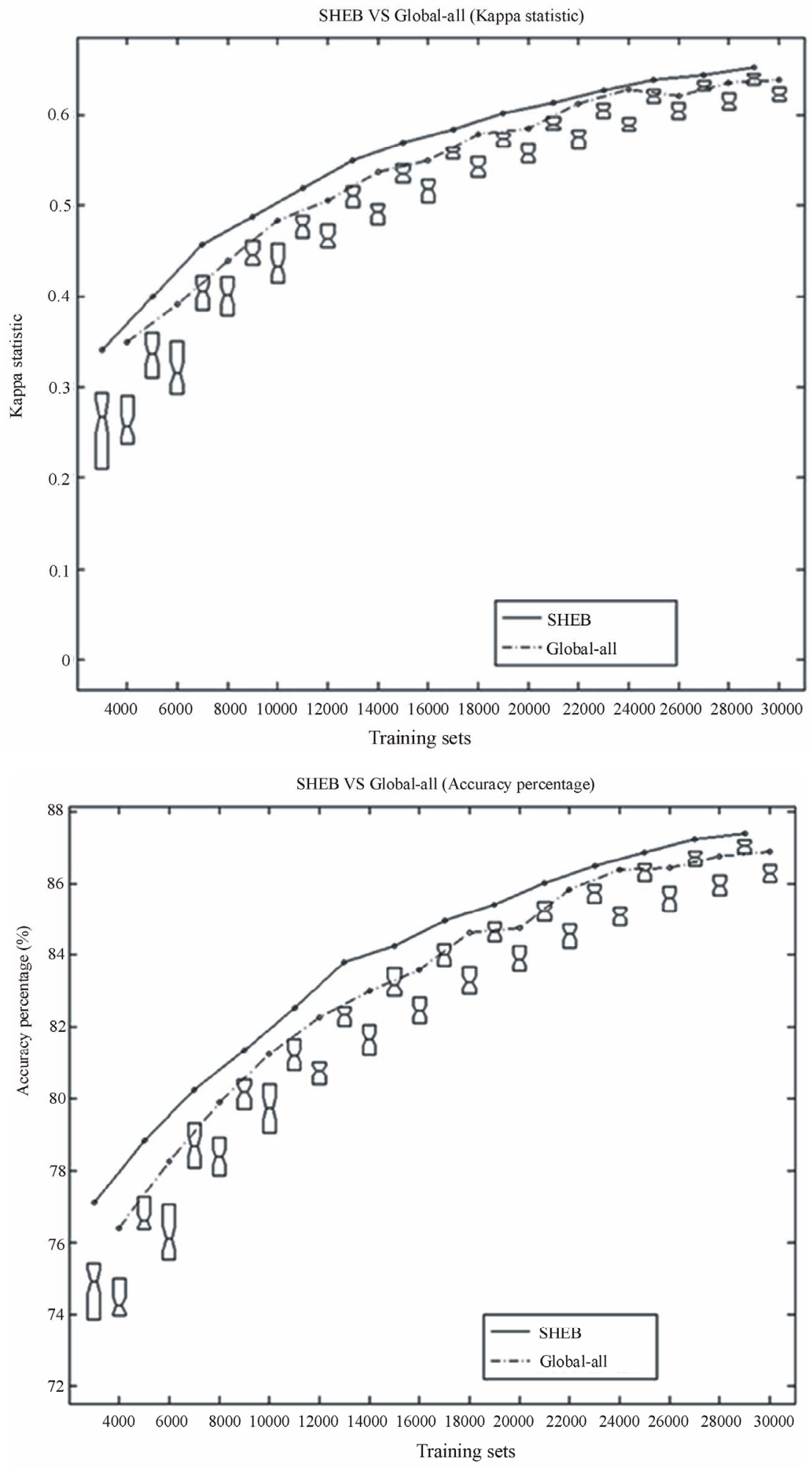

Figure 6. Prediction accuracy of the $S H E B$ model versus Global model.

plication, divides the study area into homogenous subregions, and then applies different models in each subregion. This alternative approach in urban growth model- ing produces higher accuracy results than a globally trained and applied model.

More specifically, using decision trees as the underly- 
Table 1. Student's t-test for decision trees.

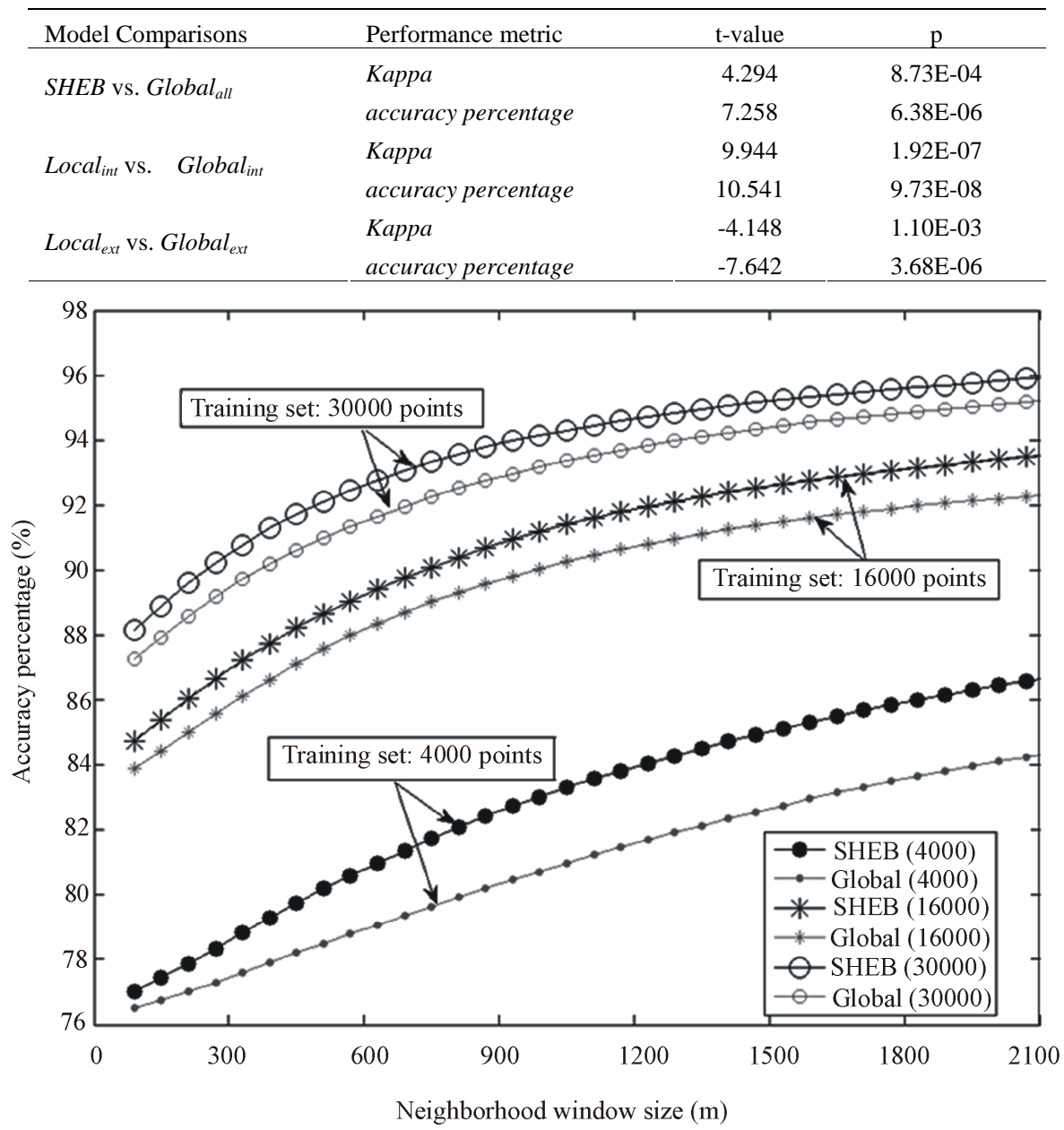

Figure 7. Multi-scale accuracy comparison between proposed (SHEB) and benchmark (Global) models using 4000,16000 and 30,000 training points.

ing algorithmic classifier, the developed local models, suitably aggregated, produce improved prediction results than a single global model. The fusion of Local Interior model ( Local $_{\text {int }}$ ) and the Global Exterior model (Global ${ }_{\text {ext }}$ ) was more accurate than a decision tree-based Global model.

Different sizes of training sets exhibited different accuracies in both Kappa and accuracy percentages. As expected, the larger the training set, the better the model accuracy performance. It is interesting to point out that the rate of accuracy improvement with increases in training size was higher for the interior model, suggesting a larger heterogeneity within that subregion. There was also a saturation point where further training sizes increases resulted in minor accuracy improvements. More specifically, the improvement in Kappa for different training sample sizes was approximately 1.0 and 1.5 (out of 100) for maximum and average values respectively. The corresponding differences of accuracy per- centages are $0.4 \%$ and $0.6 \%$ at the pixel level. A t-test comparison also supported our model selection suggesting the statistical significance of these improvements. Most importantly for urban planning purposes, this improvement reaches approximately $3 \%$ at the $1 \mathrm{~km}$ modeling scale and for small training datasets. Therefore, using the proposed methodology, we can obtain satisfactory accuracies when working in large neighbourhoods, especially when the training sample size is small. The latter is desirable for urban planners because restricted data availability is a common problem in such projects. We should note that even though the decision tree method offered significant statistical improvements, it did not exhibit any over/under performance in specific localized areas suggesting that further model segmentation may be difficult.

Incorporation of spatial heterogeneity is important for planning urban development and designing the appropriate location for establishing new facilities. The unique- 
ness of a subregion can be identified not only by characteristics on its spatial and morphological properties, but also based on socioeconomic factors which may be implicitly present in these spatial representations. An interesting future investigation could base model regionalization on socioeconomic and administrative variables. For example, different models could be based on governing units that inherently may behave differently. The local information can provide reliable modeling adaptability because expert knowledge can more easily be incorporated in homogenous subregions rather than in the entire study area. The SHEB model can sufficiently support the applicability of different homogenous subregion extractions, in order to handle the spatial heterogeneity. The usage of interior and exterior subregions in the present study acts as a proof of concept. Introducing further spatial heterogeneity into the model could potentially lead to further improvements in the prediction accuracy of urban development.

\section{Acknowledgements}

This research was supported by the National Aeronautics and Space Administration through an award for Dr. Mountrakis from the New Investigator Program (award \# NNX08AR11G).

\section{References}

[1] United Nations Population Fund, "State of the World Population 2007: Unleashing the Potential of Urban Growth,” United Nations Population Fund, United Nations Publications, 2007.

[2] United Nations Population Fund, "The State of World Population,” United Nations Population Fund, United Nations Publications, 1999.

[3] A. Rahman, Y. Kumar, S. Fazal and S. Bhaskaran, "Urbanization and Quality of Urban Environment Using Remote Sensing and GIS Techniques in East Delhi-India,” Journal of Geographic Information System, Vol. 3, No. 1, 2011, pp. 62-84. doi:10.4236/jgis.2011.31005

[4] R. M. Mendes and R. Lorandi, "Geospatial Analysis of Geotechnical Data Applied to Urban Infrastructure Planning,” Journal of Geographic Information System, Vol. 2, No. 1, 2010, pp. 23-31. doi:10.4236/jgis.2010.21006

[5] H. Briassoulis, "Analysis of Land Use Change: Theoretical and Modeling Approaches,” West Virginia University: Regional Research Institute, Morgantown, 2000. http://www.rri.wvu.edu/WebBook/Briassoulis/contents.ht m

[6] J. I. Barredo, M. Kasanko, N. Mccormick and C. Lavalle, "Modelling Dynamic Spatial Processes: Simulation of Urban Future Scenarios through Cellular Automata," Landscape and Urban Planning, Vol. 64, No. 3, 2003, pp.145-160. doi:10.1016/S0169-2046(02)00218-9
[7] Veldkamp and P. H. Verburg, "Modelling Land Use Change and Environmental Impact," Journal of environmental management, Vol. 72, No. 1-2, 2004, pp. 1-3. doi:10.1016/j.jenvman.2004.04.004

[8] M. Batty, “Agents, Cells, and Cities: New Representational Models for Simulating Multiscale Urban Dynamics," Environment and Planning A, Vol. 37, No. 8, 2005, pp. 1373-1394. doi:10.1068/a3784

[9] P. H. Verburg and A. Veldkamp, "Introduction to the Special Issue on Spatial Modeling to Explore Land Use Dynamics," International Journal of Geographical Information Science, Vol. 19, No. 2, 2005, pp. 99-102. doi:10.1080/13658810410001713362

[10] J. Wu and R. Hobbs, "Key Issues and Research Priorities in Landscape Ecology: An Idiosyncratic Synthesis," Landscape Ecology, Vol. 17, No. 4, 2002, pp. 355-365. doi:10.1023/A:1020561630963

[11] E. G. Irwin, "New Directions for Urban Economic Models of Land Use Change: Incorporating Spatial Dynamics and Heterogeneity," Journal of Regional Science, Vol. 50, No. 1, 2010, pp. 65-91. doi:10.1111/j.1467-9787.2009.00655.x

[12] J. R. Eastman, L. A. Solorzano and M. E. Van Fossen, "Transition Potential Modeling for Land-Cover Change," In: D. J. Maguire, M. Batty and M. F. Goodchild, Eds., GIS, Spatial Analysis, and Modeling, ESRI Press, Redlands, 2005, pp. 357-385.

[13] J. R. Eastman, “Idrisi Kilimanjaro, Manual," Clark Labs, Clark University, Worcester, 2003.

[14] R. Aspinall, "Modelling Land Use Change with Generalized Linear Models-A Multi-Model Analysis of Change between 1860 and 2000 in Gallatin Valley, Montana," Journal of environmental management, Vol. 72, No. 1-2, 2004, pp. 91-103. doi:10.1016/j.jenvman.2004.02.009

[15] Sebastian-Lopez, R. Salvador-Civil, J. Gonzalo-Jimenez and J. Sanmiguel-Ayanz, "Integration of Socio-Economic and Environmental Variables for Modelling Long-Term Fire Danger in Southern Europe," European Journal of Forest Research, Vol. 127, No. 2, 2008, pp. 149-163. doi:10.1007/s10342-007-0191-5

[16] J. Allen and K. Lu, "Modeling and Prediction of Future Urban Growth in the Charleston Region of South Carolina: A GIS-Based Integrated Approach,” Ecology and Society, Vol. 8, No. 2, 2003, p. 2.

[17] P. H. Verburg, T. C. M. De Nijs, J. Ritsema Van Eck, H. Visser and K. De Jong, "A Method to Analyse Neighbourhood Characteristics of land Use Patterns," Computers, Environment and Urban Systems, Vol. 28, No. 6, 2004, pp. 667-690. doi:10.1016/j.compenvurbsys.2003.07.001

[18] Z. Hu and C. P. Lo, "Modeling Urban Growth in Atlanta Using Logistic Regression,” Computers, Environment and Urban Systems, Vol. 31, No. 6, 2007, pp. 667-688. doi:10.1016/j.compenvurbsys.2006.11.001

[19] B. Huang, L. Zhang and B. Wu, "Spatiotemporal Analysis of Rural-Urban Land Conversion,” International Journal of Geographical Information Science, Vol. 23, No. 3, 
2009, pp. 379-398. doi:10.1080/13658810802119685

[20] N. H. Augustin, M. A. Mugglestone and S. T. Buckland, "An Autologistic Model for the Spatial Distribution of Wildlife,” Journal of Applied Ecology, Vol. 33, No. 2, 1996, pp. 339-347. doi:10.2307/2404755

[21] K. P. Overmars, G. H. J. De Koning and A. Veldkamp, "Spatial Autocorrelation in Multi-Scale Land Use Models,” Ecological Modelling, Vol. 164, No. 2-3, 2003, pp. 257-270. doi:10.1016/S0304-3800(03)00070-X

[22] C. F. Dormann, “Assessing the Validity of Autologistic Regression,” Ecological Modelling, Vol. 207, No. 2-4, 2007, pp. 234-242. doi:10.1016/j.ecolmodel.2007.05.002

[23] Getis and D. A. Griffith, "Comparative Spatial Filtering in Regression Analysis,” Geographical Analysis, Vol. 34, No. 2, 2002, pp. 130-140.

[24] J. Malczewski, “Ordered Weighted Averaging with Fuzzy Quantifiers: GIS-Based Multicriteria Evaluation for Land-Use Suitability Analysis," International Journal of Applied Earth Observation and Geoinformation, Vol. 8, No. 4, 2006, pp. 270-277. doi:10.1016/j.jag.2006.01.003

[25] Gemitzi, V. A. Tsihrintzis, E. Voudrias, C. Petalas and G. Stravodimos, "Combining Geographic Information System, Multicriteria Evaluation Techniques and Fuzzy Logic in Siting MSW Landfills,” Environmental Geology, Vol. 51, No. 5, 2007, pp. 797-811. doi:10.1007/s00254-006-0359-1

[26] M. Zarghami and F. Szidarovszky, "Fuzzy Quantifiers in Sensitivity Analysis of OWA Operator," Computers and Industrial Engineering, Vol. 54, No. 4, 2008, pp. 1006-1018. doi:10.1016/j.cie.2007.11.012

[27] Q. Yang, X. Li and X. Shi, "Cellular Automata for Simulating Land Use Changes Based on Support Vector Machines," Computers and Geosciences, Vol. 34, No. 6, 2008, pp. 592-602. doi:10.1016/j.cageo.2007.08.003

[28] C. Huang, K. Song, S. Kim, J. R. G. Townshend and P. Davis, "Use of a Dark Object Concept and Support Vector Machines to Automate Forest Cover Change Analysis,” Remote Sensing of Environment, Vol. 112, No. 3, 2008, pp. 970-985. doi:10.1016/j.rse.2007.07.023

[29] T. Kuemmerle, P. Hostert, V. C. Radeloff, S. Van der Linden and K. Perzanowski, "Cross-Border Comparison of Post-Socialist Farmland Abandonment in the Carpathians," Ecosystems, Vol. 11, No. 4, 2008, pp. 614-628. doi:10.1007/s10021-008-9146-Z

[30] D. E. Goldberg, "Neighborhood Competition in an Old-Field Plant Community,” Ecology, Vol. 68, No. 5, 1987, pp. 1211-1223. doi:10.2307/1939205

[31] P. J. Burton, "Some Limitations Inherent to Static Indices of Plant Competition," Canadian Journal of Forest Research, Vol. 23, No. 10, 1993, pp. 2141-2152. doi:10.1139/x93-267

[32] W. D'amato and K. J. Puettmann, “The Relative Dominance Hypothesis Explains Interaction Dynamics in Mixed Species Alnus Rubra/Pseudotsuga Menziesii Stands," Journal of Ecology, Vol. 92, No. 3, 2004, pp. 450-463. doi:10.1111/j.0022-0477.2004.00888.x

[33] S. W. Simard and B. J. Zimonick, "Neighborhood Size
Effects on Mortality, Growth and Crown Morphology of Paper Birch,” Forest Ecology and Management, Vol. 214, No. 1-3, 2005, pp. 251-265. doi:10.1016/j.foreco.2005.04.009

[34] C. He, N. Okada, Q. Zhang, P. Shi and J. Li, "Modelling Dynamic Urban Expansion Processes Incorporating a Potential Model with Cellular Automata,” Landscape and Urban Planning, Vol. 86, No. 1, 2008, pp. 79-91. doi:10.1016/j.landurbplan.2007.12.010

[35] S. J. Walsh, J. P. Messina, C. F. Mena, G. P. Malanson and P. H. Page, "Complexity Theory, Spatial Simulation Models, and Land Use Dynamics in the Northern Ecuadorian Amazon,” Geoforum, Vol. 39, No. 2, 2008, pp. 867-878. doi:10.1016/j.geoforum.2007.02.011

[36] Menard and D. J. Marceau, "Simulating the Impact of Forest Management Scenarios in an Agricultural Landscape of Southern Quebec, Canada, Using a Geographic Cellular Automata," Landscape and Urban Planning, Vol. 79, No. 3-4, 2007, pp. 253-265. doi:10.1016/j.landurbplan.2006.02.016

[37] H. Couclelis, "From Cellular Automata to Urban Models: New Principles for Model Development and Implementation,” Environment and Planning B: Planning and Design, Vol. 24, No. 2, 1997, pp. 165-174. doi: $10.1068 / \mathrm{b} 240165$

[38] R. White and G. Engelen, "High-Resolution Integrated Modelling of the Spatial Dynamics of Urban and Regional Systems," Computers, Environment and Urban Systems, Vol. 24, No. 5, 2000, pp. 383-400. doi:10.1016/S0198-9715(00)00012-0

[39] X. Liu, X. Li, L. Liu, J. He and B. Ai, “A Bottom-Up Approach to Discover Transition Rules of Cellular Automata Using Ant Intelligence," International Journal of Geographical Information Science, Vol. 22, No. 11-12, 2008, pp. 1247-1269. doi:10.1080/13658810701757510

[40] J. V. Vliet, R. White and S. Dragicevic, "Modeling Urban Growth Using a Variable Grid Cellular Automaton," Computers, Environment and Urban Systems, Vol. 33, No. 1, 2009, pp. 35-43. doi:10.1016/j.compenvurbsys.2008.06.006

[41] F. Del Frate, F. Pacifici, G. Schiavon and C. Solimini, "Use of Neural Networks for Automatic Classification from High-Resolution Images," IEEE Transactions on Geoscience and Remote Sensing, Vol. 45, No. 4, 2007, pp. 800-809. doi:10.1109/TGRS.2007.892009

[42] M. J. Canty, "Boosting a Fast Neural Network for Supervised Land Cover Classification,” Computers and Geosciences, Vol. 35, No. 6, 2009, pp. 1280-1295. doi:10.1016/j.cageo.2008.07.004

[43] T. Kavzoglu, "Increasing the Accuracy of Neural Network Classification Using Refined Training Data," Environmental Modelling Software, Vol. 24, No. 7, 2009, pp. 850. doi:10.1016/j.envsoft.2008.11.012

[44] JR. R. G. Pontius, W. Boersma, J. Castella, K. Clarke and T. Nijs, "Comparing the input, output, and Validation Maps for Several Models of Land Change," Annals of Regional Science, Vol. 42, No. 1, 2008, pp. 11-37. doi:10.1007/s00168-007-0138-2 
[45] T. Lakes, D. Muller and C. Kruger, "Cropland Change in Southern Romania: A Comparison of Logistic Regressions and Artificial Neural Networks," Landscape Ecology, Vol. 24, No. 9, 2009, pp. 1195-1206. doi:10.1007/s10980-009-9404-2

[46] B. C. Pijanowski, A. Tayyebi, M. R. Delavar and M. J. Yazdanpanah, "Urban Expansion Simulation Using Geospatial Information System and Artificial Neural Networks,” International Journal of Environmental Research, Vol. 3, No. 4, 2009, pp. 493-502.

[47] B. C. Pijanowski, D. G. Brown, B. A. Shellito and G. A. Manik, "Using Neural Networks and GIS to Forecast Land Use Changes: A Land Transformation Model," Computers, Environment and Urban Systems, Vol. 26, No. 6, 2002, pp. 553-575. doi:10.1016/S0198-9715(01)00015-1

[48] C. E. Rizkalla and R. K. Swihart, "Forecasting the Effects of Land-Use Change on Forest Rodents in Indiana," Environmental Management, Vol. 44, No. 5, 2009, pp. 899-908.

[49] W. Liu and K. C. Seto, "Using the ART-MMAP neural Network to Model and Predict Urban Growth: A Spatiotemporal Data Mining Approach," Environment and Planning B: Planning and Design, Vol. 35, No. 2, 2008, pp. 296-317. doi:10.1068/b3312

[50] X. Li and A. G. Yeh, "Calibration of Cellular Automata by Using Neural Networks for the Simulation of Complex Urban Systems," Environment and Planning A, Vol. 33, No. 8, 2001, pp. 1445-1462. doi:10.1068/a33210

[51] Y. Mahajan and P. Venkatachalam, "Neural Network Based Cellular Automata Model for Dynamic Spatial Modeling in GIS," Lecture Notes in Computer Science (Including Subseries Lecture Notes in Artificial Intelligence and Lecture Notes in Bioinformatics), Vol. 5592, Part 1, 2009, pp. 341-352.

[52] B. C. Pijanowski, S. Pithadia, B. A. Shellito and K. Alexandridis, "Calibrating a Neural Network-Based Urban Change Model for Two Metropolitan Areas of the Upper Midwest of the United States," International Journal of Geographical Information Science, Vol. 19, No. 2, 2005, pp. 97-215. doi:10.1080/13658810410001713416

[53] Q. Guan, L. Wang and K. C. Clarke, "An ArtificialNeural-Network-Based, Constrained CA Model for Simulating Urban Growth," Cartography and Geographic Information Science, Vol. 32, No. 4, 2005, pp. 369-380. doi:10.1559/152304005775194746

[54] C. M. Almeida, J. M. Gleriani, E. F. Castejon and B. S. Soares-Filho, "Using Neural Networks and Cellular Automata for Modelling Intra-Urban Land-Use Dynamics,” International Journal of Geographical Information Science, Vol. 22, No. 9, 2008, pp. 943-963.

[55] J. C. Chan, K. Chan and A. G. Yeh, "Detecting the Nature of Change in an Urban Environment: A Comparison of Machine Learning Algorithms," Photogrammetric Engineering and Remote Sensing, Vol. 67, No. 2, 2001, pp. 213-225.

[56] X. Li and C. Claramunt, "A Spatial Entropy-Based Decision Tree for Classification of Geographical Informa- tion,” Transactions in GIS, Vol. 10, No. 3, 2006, pp. 451-467. doi:10.1111/j.1467-9671.2006.01006.X

[57] X. Liu, X. Li, X. Shi, S. Wu and T. Liu, "Simulating Complex Urban Development Using Kernel-Based NonLinear Cellular Automata,” Ecological Modelling, Vol. 211, No. 1-2, 2008, pp. 169-181. doi:10.1016/j.ecolmodel.2007.08.024

[58] X. Li and A. G. Yeh, “Analyzing Spatial Restructuring of Land Use Patterns in a Fast Growing Region Using Remote Sensing and GIS,” Landscape and Urban Planning, Vol. 69, No. 4, 2004, pp. 335-354. doi:10.1016/j.landurbplan.2003.10.033

[59] S. E. Sesnie, P. E. Gessler, B. Finegan and S. Thessler, "Integrating Landsat TM and SRTM-DEM Derived Variables with Decision Trees for Habitat Classification and Change Detection in Complex Neotropical Environments," Remote Sensing of Environment, Vol. 112, No. 5, 2008, pp. 2145-2159. doi:10.1016/j.rse.2007.08.025

[60] J. R. Quinlan, “Probabilistic Decision Tress,” In: K. Yves and R. Michalski, Eds., Machine Learning: An Artificial Intelligence Approach, Morgan Kaufmann, San Mateo, Vol. 3, 1983, pp. 140-152.

[61] R. I. McDonald and D. L. Urban, "Spatially Varying Rules of Landscape Change: Lessons from a Case Study," Landscape and Urban Planning, Vol. 74, No. 1, 2006, pp. 7-20. doi:10.1016/j.landurbplan.2004.08.005

[62] J. Liu and W. W. Taylor, "Integrating Landscape Ecology into Natural Resource Management,” Cambridge University Press, Cambridge, 2002. doi:10.1017/CBO9780511613654

[63] D. G. Brown, S. Page, R. Riolo, M. Zellner and W. Rand, "Path Dependence and the Validation of Agent-Based Spatial Models of Land Use," International Journal of Geographical Information Science, Vol. 19, No. 2, 2005, pp. 153-174. doi:10.1080/13658810410001713399

[64] J. Wang and G. Mountrakis, "Developing a Multi-Network Urbanization (MuNU) Model: A Case Study of Urban Growth in Denver, Colorado,” International Journal of Geographical Information Science, Vol. 25, No. 2, 2011, pp. 229-253. doi:10.1080/13658810903473213

[65] M. Raty and A. Kangas, "Localizing General Models with Classification and Regression Trees,” Scandinavian Journal of Forest Research, Vol. 23, No. 5, 2008, pp. 419-430. doi:10.1080/02827580802378826

[66] M. Herold, H. Couclelis and K. C. Clarke, "The Role of Spatial Metrics in the Analysis and Modeling of Urban Land Use Change," Computers, Environment and Urban Systems, Vol. 29, No. 4, 2005, pp. 369-399. doi:10.1016/j.compenvurbsys.2003.12.001

[67] L. Porter-Bolland, E. A. Ellis and H. L. Gholz, "Land Use Dynamics and Landscape History in La Montaña, Campeche, Mexico,” Landscape and Urban Planning, Vol. 82, No. 4, 2007, pp. 198-207.

[68] J. S. Deng, K. Wang, Y, Hong and J. G. Qi, "SpatioTemporal Dynamics and Evolution of Land Use Change and Landscape Pattern in Response to Rapid Urbanization,” Landscape and Urban Planning, Vol. 92, No. 3-4, 2009, pp. 187-198. 
doi:10.1016/j.landurbplan.2009.05.001

[69] K. McGarigal, S. Tagil and S. A. Cushman, "Surface Metrics: An Alternative to Patch Metrics for the Quantification of Landscape Structure," Landscape Ecology, Vol. 24, No. 3, 2009, pp. 433-450. doi:10.1007/s10980-009-9327-y

[70] D. Triantakonstantis and S. Barr, "A Spatial Structural and Statistical Approach to Building Classification of Residential Function for City-Scale Impact Assessment Studies," Lecture Notes in Computer Science, Vol. 5592, 2009, pp. 221-236. doi:10.1007/978-3-642-02454-2_16

[71] Lagarias, "Fractal Analysis of the Urbanization at the Outskirts of the City: Models, Measurement and Explanation," CyberGeo: European Journal of Geography, 2007, pp. 1-16.

[72] S. Barr and M. Barnsley, “A Region-Based, Graph-Theoretic Data Model for the Inference of Second-Order Thematic Information from Remotely-Sensed Images," International Journal of Geographical Information Science, Vol. 11, No. 6, 1997, pp. 555-576. doi:10.1080/136588197242194

[73] K. Wu, N. Nunan, J. W. Crawford, I. M. Young and K. Ritz, "An Efficient Markov Chain Model for the Simulation of Heterogeneous Soil Structure," Soil Science Society of America Journal, Vol. 68, No. 2. 2004, pp. 346-351. doi:10.2136/sssaj2004.0346
[74] J. Gong, Y. Liu and B. Xia, "Spatial Heterogeneity of Urban Land-Cover Landscape in Guangzhou from 1990 to 2005,” Journal of Geographical Sciences, Vol. 19, No. 2, 2009, pp. 213-224. doi:10.1007/s11442-009-0213-y

[75] T. G. Wade, J. D. Wickham, N. Zacarelli and K. H. Riitters, "A Multi-Scale Method of Mapping Urban Influence,” Environmental Modelling \& Software, Vol. 24, No. 10, 2009, pp. 1252-1256. doi:10.1016/j.envsoft.2009.03.006

[76] M. Story and R. G. Congalton, “Accuracy Assessment: A User's Perspective,” Photogrammetric Engineering and Remote Sensing, Vol. 52, No. 3, 1986, pp. 397-399.

[77] R. G. Congalton, "A Review of Assessing the Accuracy of Classifications of Remotely Sensed Data," Remote Sensing of Environment, Vol. 37, No. 1, 1991, pp. 35-46. doi:10.1016/0034-4257(91)90048-B

[78] J. Cohen, “A Coefficient of Agreement for Nominal Scales,” Educational and Psychological Measurement, Vol. 20, No. 1, 1960, pp. 37-46. doi: $10.1177 / 001316446002000104$

[79] R. Costanza, "Model Goodness of Fit: A Multiple Resolution Procedure," Ecological Modelling, Vol. 47, No. 3-4, 1989, pp. 199-215. doi:10.1016/0304-3800(89)90001-X 